\title{
Binding proteins internalized by PTD-fused ligands allow the intracellular sequestration of selected targets by ligand exchange
}

\author{
MARKUS A. MOOSMEIER ${ }^{1,2}$, JULIA BULKESCHER ${ }^{1}$, KARIN HOPPE-SEYLER ${ }^{1}$ and FELIX HOPPE-SEYLER ${ }^{1}$ \\ ${ }^{1}$ Molecular Therapy of Virus-Associated Cancers (F065), German Cancer Research Center, \\ Im Neuenheimer Feld 242, D-69120 Heidelberg, Germany
}

Received November 3, 2009; Accepted November 27, 2009

\section{DOI: 10.3892/ijmm_00000377}

\begin{abstract}
The targeted inactivation of intracellular molecules has important therapeutic potential. For this purpose, it could be envisioned to introduce specifically designed binding proteins into cells by covalent linkage to protein transduction domains (PTDs). However, stable linkage of a PTD to a cargo may affect its conformation and, hence, its binding property inside the cell. Here, we analyzed the ability of non-covalently linked PTDs to internalize the model binding proteins streptavidin (SA) and Strep-Tactin (ST). Notably, inside the cell, the PTD-Strep-tag II ligand used for internalization of SA was displaced by the model target biotin which exhibits a higher binding affinity for the same binding pocket. Thus, specifically designed binding proteins can be internalized into cells by non-covalent binding to a PTD and subsequently be used for capturing given intracellular target molecules by ligand exchange. Under therapeutic aspects, it could be envisioned to further develop such systems for the intracellular sequestration, and consequently, functional inactivation of pathologically relevant factors.
\end{abstract}

\section{Introduction}

Many therapeutic agents prescribed today belong to the group of cell-permeable small molecules which are derived from organics and peptides. In general, small molecules can enter the cell by simple diffusion and interact with intracellular targets in defined ways, for instance by blocking the target's active site with sufficient affinity or by irreversible covalent binding. However, many small molecules suffer from lack of specificity, leading to unwanted side effects and toxicity.

Macromolecular therapeutic agents based on proteins have several hypothetical advantages over small molecules

Correspondence to: Dr Markus A. Moosmeier, ${ }^{2}$ Present address: MorphoSys AG, Lena-Christ-Str. 48, D-82152 Martinsried/Planegg, Germany

E-mail: markus.moosmeier@morphosys.com

Key words: streptavidin, protein transduction, therapy
(1). Firstly, they possess a highly specific and complex set of functions that cannot be mimicked by small molecules. Secondly, since proteins target molecules in a highly specific way, they often have less potential to induce unspecific side effects than small molecules which can bind to similarly shaped pockets of proteins other than the target. Thirdly, protein therapeutics can provide effective replacement treatment for diseases in which proteins are mutated, truncated, or not expressed. On the other hand, however, most of the macromolecular therapeutic agents [e.g., antibodies (2), their fragments (3), or other proteins with specific binding potential (4), such as anticalins (5)] cannot penetrate cellular membranes of intact cells due to their biophysical properties (e.g., size, charge, and polarity). This restricts their use to extracellular proteins or to ectodomains of cell-surface receptors. Nevertheless, due to their potential advantages over small molecules, there has been growing interest in the use of therapeutic macromolecular agents against intracellular targets.

One way to induce cell membrane permeability of proteins is their covalent fusion to protein transduction domains (PTDs). PTDs are short peptides which enable internalization of linked molecules into a variety of eukaryotic cell types. An example of a well-studied cationic PTD is the Tat13 peptide (YGRKKRRQRRRPP) which is derived from the HIV-1 trans-activator protein (6-10). Since the discovery of PTDs in $1988(6,7)$, a broad range of bioactive proteins has been delivered into cells by this method, both in vitro and in vivo (11). This technology is thus considered to bear enormous potential to introduce macromolecular therapeutics into cells (12-14). Notably, PTDs may also penetrate intact epidermis and dermis which could enable transdermal delivery of cargos upon topical application (15-17).

However, the genetic fusion of PTDs to proteins can result in reduced protein expression and purification levels (10). Moreover, the stable linkage of PTDs, either by genetic or chemical means, can impair the biophysical properties of the cargo (10). Therefore, attempts have been made to generate universal cell permeable transporters that allow the concomitant internalization of cargos by non-covalent binding. Streptavidin (SA), an extracellular homotetrameric protein secreted by the bacterium Streptomyces avidinii (18), or SA fusion proteins were delivered into cells by PTD-biotin $(19,20)$, on the basis of the high affinity of the SA/biotin 
interaction $(21,22)$. The same SA binding pocket targeted by biotin is also reversibly bound by designed peptide motifs, such as Strep-tag II (WSHPQFEK) (23). In addition, Streptag II exhibits intrinsic affinity towards Strep-Tactin (ST), a derivative of SA (24). This Strep-tag II/ST system has become very common for one-step purification of proteins or high-affinity detection (23).

Here, we aimed to develop a novel two-component delivery system for the internalization of non-modified binding molecules (Fig. 1). The model system consists of the noncell permeable SA protein containing a binding pocket for Strep-tag II which, in turn, is fused to a PTD. The same SA pocket is also bound by intracellular biotin, but with higher affinity. We hypothesized that, firstly, the PTD-Streptag II ligand should allow the cellular internalization of SA. Secondly, inside the cell, the PTD-Strep-tag II ligand should then be displaced by biotin. In principle, such systems could allow the sequestration and, consequently, functional inactivation of specific intracellular target molecules.

\section{Materials and methods}

Construction, expression, and purification of $S A, S T$, and Tat13-SA. The pET-21a plasmid (Novagen, Darmstadt, Germany) encoding core SA was kindly provided by P.S. Stayton. ST was expressed from the same vector backbone after QuikChange mutagenesis (Stratagene, Heidelberg, Germany) of the SA portion with primers 5'-G ACC GGT ACC TAC ATC GGT GCG AGG GGT AAC GCT GAA TC-3' and 5'-GA TTC AGC GTT ACC CCT CGC ACC GAT GTA GGT ACC GGT C-3' [bold letters indicate mutations to induce amino acid substitutions $E^{44} \mathrm{I}, \mathrm{S}^{45} \mathrm{G}$, and $\mathrm{V}^{47} \mathrm{R}$ which convert SA into ST (24)]. Tat13-SA was generated by PCR-amplification using Taq DNA polymerase (Invitrogen, Karlsruhe, Germany), the forward primer 5'-G GAA TTC CAT ATG TAC GGA AGA AAG AAG CGC AGA CAA AGA AGA CGT CCA CCA GGT GCT GAA GCT GGT ATC ACC GGC ACC-3' (bold letters denote the respective Tat13 PTD sequence), and reverse primer 5'-CGC AAG CTT TTA TTA GGA AGC AGC GG-3'. PCR-products were digested with $N d e I$ and HindIII (New England Biolabs, Frankfurt, Germany) and subsequently ligated into linearized pET-21a. Correct sequences of the inserts were confirmed by DNA sequencing.

SA, ST, and Tat13-SA proteins were expressed in BL21(DE3) (Stratagene) as cytoplasmic insoluble inclusion bodies that were harvested, solubilised, refolded, and purified by fractionated ammonium sulfate precipitation (25). Proteins were finally dissolved in PBS buffer and stored at $-80^{\circ} \mathrm{C}$. Purified proteins were analyzed by sodium dodecyl sulfate polyacrylamide gel electrophoresis (SDS-PAGE), matrix-assisted laser desorption/ionization time of flight mass spectrometry (MALDI-TOF MS), Edman-sequencing, and circular dichroism (CD) spectroscopy (data not shown).

Peptide syntheses. Tat13- $\mathrm{PEO}_{3}$-biotin and Tat13-Strep-tag II peptides were chemically synthesized at the Peptide Synthesis Core Facility of the German Cancer Research Center (Heidelberg, Germany) using Fmoc (N-(9-fluorenyl)methoxycarbonyl) chemistry. Crude peptides were purified by $\mathrm{C}_{18}$ reversed phase high-performance liquid chromatography (RP-HPLC) and analyzed by MS. Peptide stocks in DMSO (Merck, Darmstadt, Germany) were stored at $-80^{\circ} \mathrm{C}$ and freshly diluted in $\mathrm{H}_{2} \mathrm{O}$.

Cell culture and transduction. HeLa cells were cultured in Dulbecco's minimal essential medium (DMEM, Gibco, Eggenstein, Germany), supplemented with $10 \%$ fetal bovine serum (FBS, PAA Laboratories, Pasching, Austria), 1\% penicillin streptomycin sulfate, and $1 \%$ of $200 \mathrm{mM}$ L-glutamine (Sigma-Aldrich, Taufkirchen, Germany), at $37^{\circ} \mathrm{C}$ in $5 \%$ carbon dioxide $\left(\mathrm{CO}_{2}\right)$ atmosphere. SA, ST, SA-horseradish peroxidase (HRP, Pierce, Rockford, IL, USA), or ST-HRP (IBA, Göttingen, Germany) proteins were complexed with biotin (Supelco, Bellefonte, PA, USA), Strep-tag II (IBA), Tat13-PEO 3 -biotin, or Tat13-Strep-tag II by incubation for $15 \mathrm{~min}$ at room temperature. The complexes were directly injected into FBS-free DMEM medium.

Thermal tetramer stability. Purified SA/ST proteins $(3 \mu \mathrm{g})$ without or with a 2-fold molar excess of biotin, or Strep-tag II, were combined with SDS-containing sample buffer and heated at selected temperatures for $5 \mathrm{~min}$, then chilled on ice, and subsequently analyzed by SDS-PAGE. Proteins were stained with Coomassie brilliant blue (Serva, Heidelberg, Germany).

Western blotting. Protein extracts $(2 \mu \mathrm{g})$ were combined with SDS-containing sample buffer and boiled at 85 and $99^{\circ} \mathrm{C}$ for 5 min, separated by $15 \%$ SDS-PAGE, transferred in a semidry blotter system (cti, Idstein, Germany) to an Immobilon-P membrane (Millipore, Billerica, MA, USA), and analyzed by enhanced chemiluminescence (GE Healthcare, Munich, Germany). The following antibodies were used: rabbit antiSA antibody (Sigma-Aldrich, 1:2000), mouse anti-tubulin antibody CP06 (Calbiochem, Schwalbach, Germany, 1:5000), and anti-mouse and anti-rabbit HRP-labeled secondary antibodies (Promega, Mannheim, Germany, 1:3000).

Fluorescence microscopy. HeLa cells were plated on $35 \mathrm{~mm}$ dishes (Greiner Bio-one, Frickenhausen, Germany) at 40-60\% confluency. SA/ST proteins $(10 \mu \mathrm{M})$ were complexed with $10 \mu \mathrm{M}$ Tat13-PEO ${ }_{3}$-biotin or Tat13-Strep-tag II and directly injected into FBS-free media. After a 120-min incubation at $37^{\circ} \mathrm{C}, 5 \% \mathrm{CO}_{2}$, the cells were washed, trypsinized, plated on glass cover-slips, and fixed with $4 \%$ paraformaldehyde (Merck, Darmstadt, Germany) after 4 h. Cover-slips were stored at $-20^{\circ} \mathrm{C}$ in $70 \%$ ethanol. Internalized SA and ST proteins were detected using a rabbit anti-SA antibody (1:500) and an anti-rabbit Cy3-labeled secondary antibody (Dianova, Hamburg, Germany, 1:400). Cy3-signals were detected using a Vanox-T fluorescence microscope (Olympus, Hamburg, Germany) and an F-View camera (Olympus).

HRP assay. HeLa cells were plated on $35 \mathrm{~mm}$ dishes at 80-90\% confluency and treated with $1 \mu \mathrm{M}$ SA-HRP or ST-HRP complexed with $2 \mu \mathrm{M}$ Tat13-PEO${ }_{3}$-biotin or Tat13-Strep-tag II. Cells were incubated at $37^{\circ} \mathrm{C}, 5 \% \mathrm{CO}_{2}$ for $2 \mathrm{~h}$, then trypsinized, washed with $1 \mathrm{X}$ PBS, and lysed with $400 \mu 11 \mathrm{X}$ reporter lysis buffer (RLB, Promega). Cell lysates were incubated at room temperature for $15 \mathrm{~min}$. After vortexing and pelleting, the supernatants were stored at $-80^{\circ} \mathrm{C}$. Extracts were diluted and 


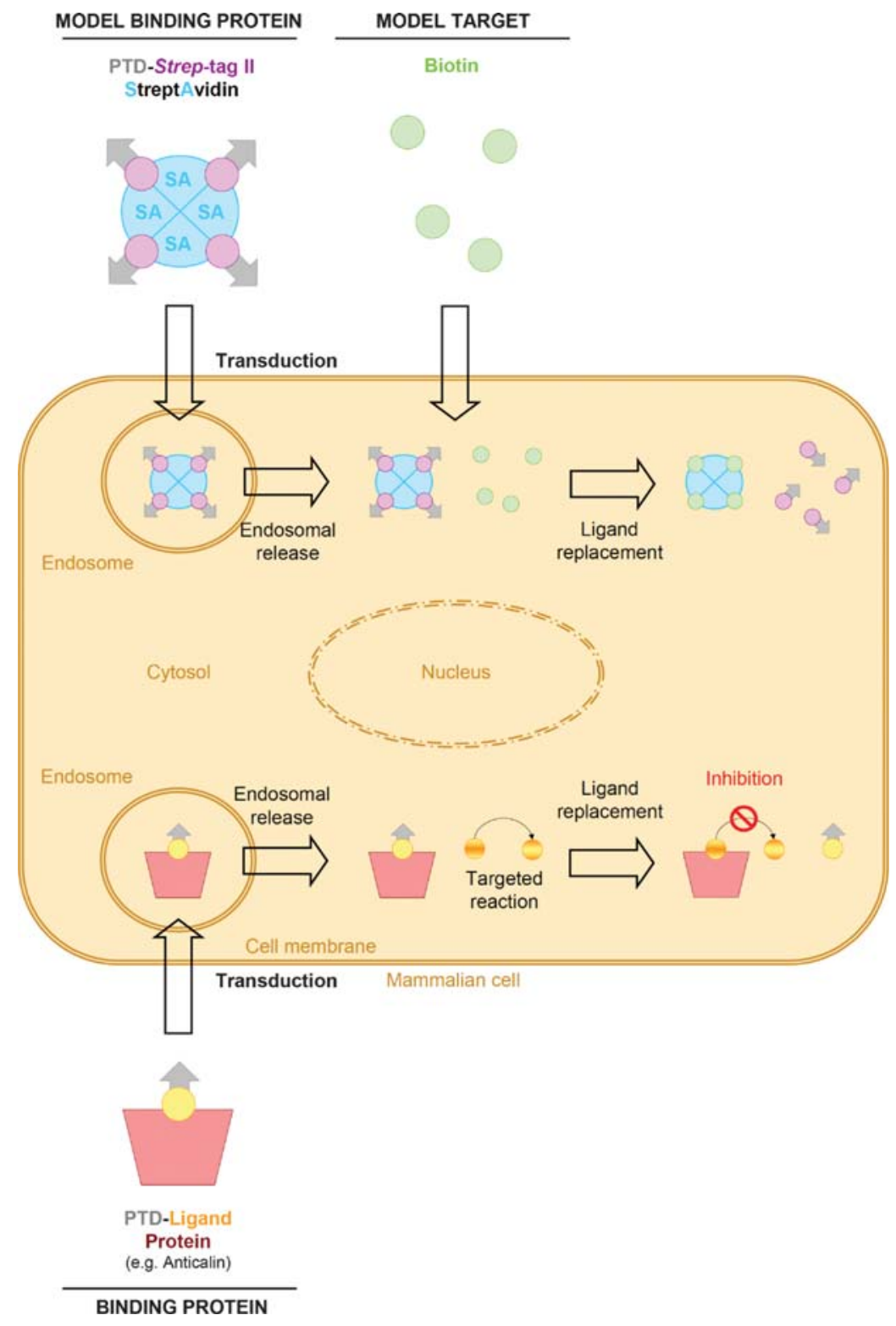

Figure 1. Model for the internalization of SA by PTD-fused Strep-tag II (upper part of the panel). SA is internalized into mammalian cells most likely via the endosomal route. In the cytoplasm, PTD-Strep-tag II is replaced by the higher affinity ligand biotin whose internalization is supported by means of the multivitamin transporter. If this system is principally functional, one could envision the application of therapeutically useful binding molecules, e.g., anticalins, using low affinity PTD-ligands for internalization (lower part of the panel). Subsequently, this ligand will be replaced by a higher affinity intracellular target molecule, leading to the functional inactivation of the latter by sequestration.

$40 \mu 1$ thereof were mixed with $10 \mu 11 \mathrm{X}$ RLB. At the same time, and under identical conditions, calibration curves were generated using a series of dilutions of SA-HRP or ST-HRP. SA-HRP and ST-HRP fusion proteins were diluted and $10 \mu \mathrm{l}$ thereof were supplemented with $40 \mu 1$ of dilutions of untreated HeLa lysates. Colorimetric reactions were initiated by adding $50 \mu 1$ of 1-Step Ultra TMB-ELISA substrate solution (Pierce). The reaction was stopped by adding $50 \mu 1$ of $2 \mathrm{M}$ sulfuric acid (Merck) and the absorbance was measured at $450 \mathrm{~nm}$. Calibration curves were fitted using the four parameter logistic (4PL) equation in SigmaPlot 10.0 (Systat Software, Erkrath, Germany). The absorbance was recalculated to the amount of internalized HRP and normalized to the amount of total protein determined by Bradford protein assay as described (26).

\section{Results}

Intracellular distribution of SA and ST internalized by Tat13-fused ligands. To investigate the uptake and intracellular distribution of model proteins by non-covalent PTD linkage, we analyzed SA and the SA-derivate ST, upon non-covalent linkage to different ligands fused to the Tat13 PTD.

HeLa cells were incubated with $10 \mu \mathrm{M}$ SA pre-complexed with $10 \mu \mathrm{M}$ Tat13-PEO - -biotin. Non-complexed SA served as negative control. After $2 \mathrm{~h}$ of incubation, cells were trypsinized to remove extracellularly attached proteins, plated on glass coverslips for fixation, and subsequently analyzed by immunofluorescence. It was found that Tat13- $\mathrm{PEO}_{3}$-biotin internalized its cargo SA into virtually $100 \%$ of the HeLa cells (Fig. 2A). 


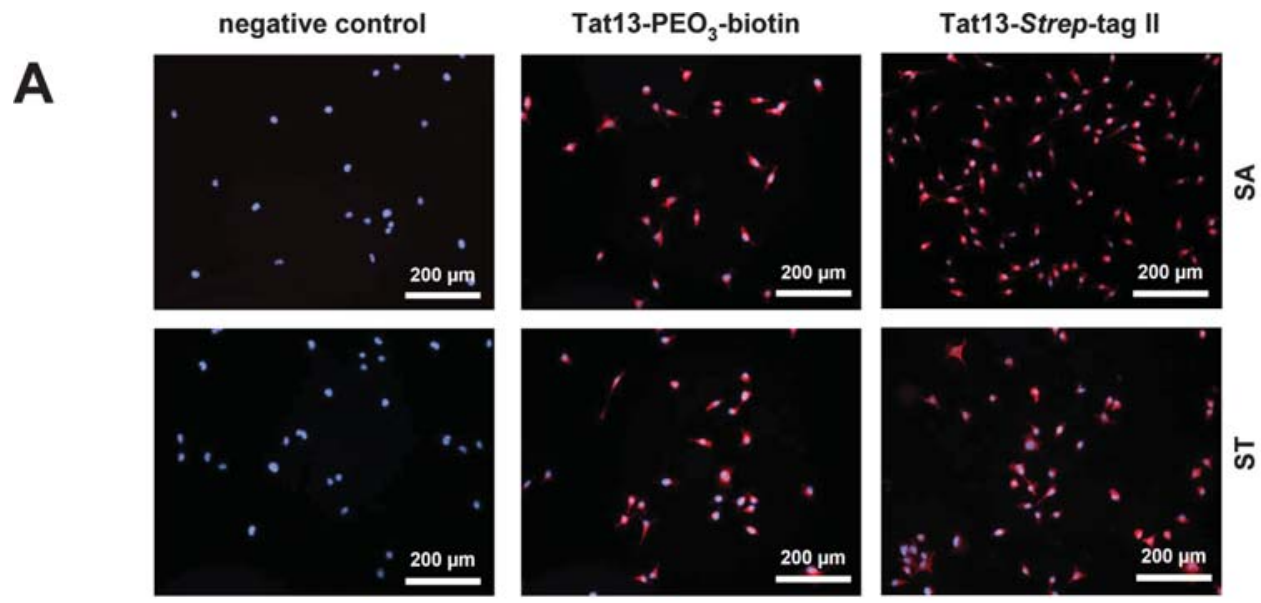

B
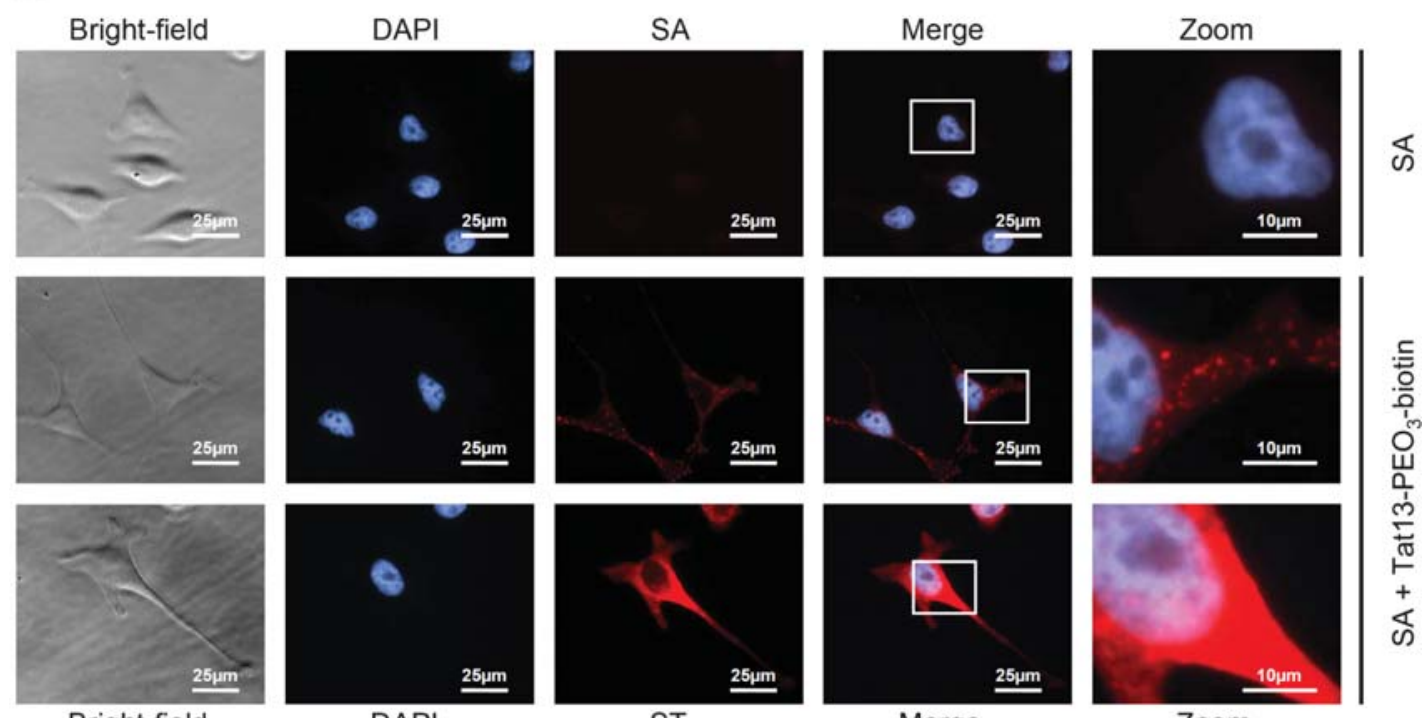

ふ
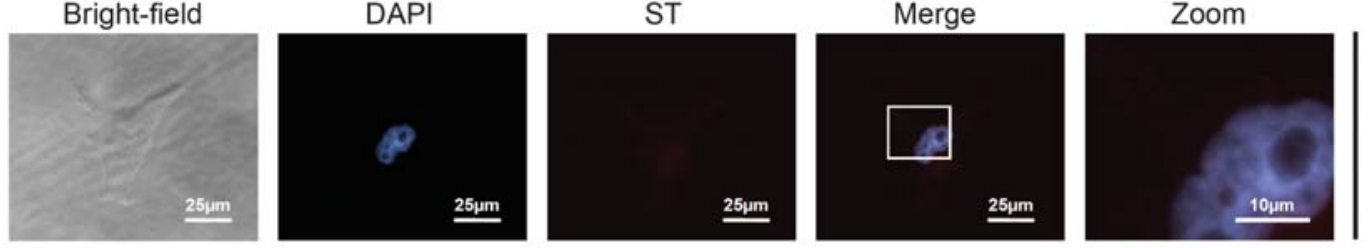

战
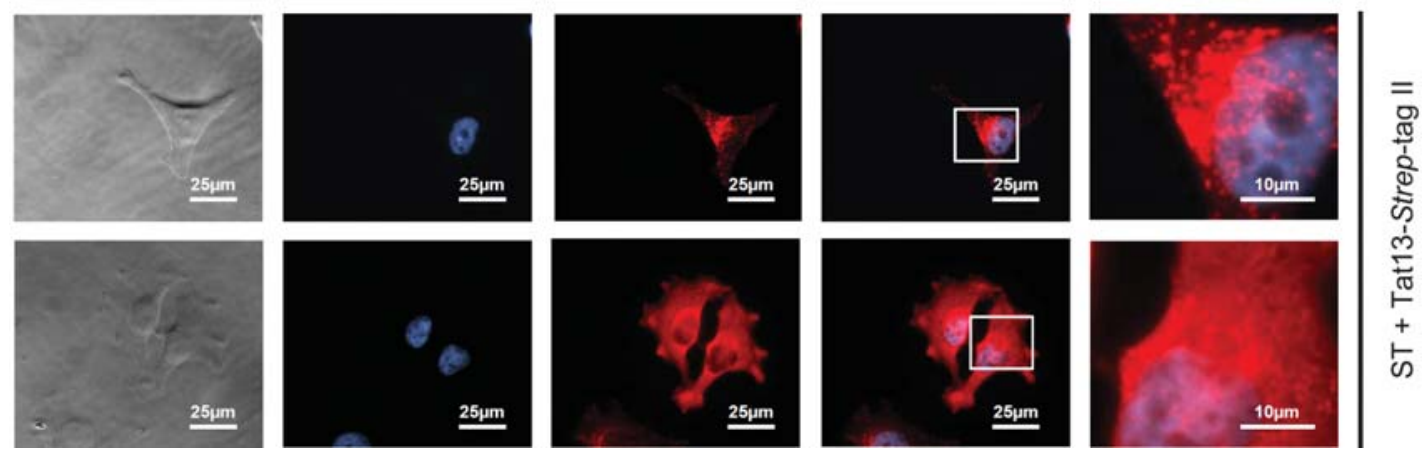

Figure 2. Immunofluorescence studies analyzing SA and ST internalization. (A) Uptake of SA or ST mediated by the Tat13-fused ligands biotin or Strep-tag II. Negative control, treatment of the cells with SA and ST in the absence of PTD-fused ligands. SA and ST were detected using an anti-SA antibody, DNA was stained with DAPI. (B) Larger magnifications to assess the intracellular distribution. SA and ST proteins internalized by PTD-fused ligands exhibited either a punctuated pattern around the cell nucleus or diffuse cytosolic staining. Zoom, higher resolution of boxed areas.

In contrast, control cells treated with $\mathrm{SA}$ in the absence of Tat13- $\mathrm{PEO}_{3}$-biotin showed no internalization. Higher resolution microscopy analyses showed that Tat13-internalized SA was typically detected in two major intracellular distribution patterns (Fig. 2B), either punctuated around the cell nucleus and/or solubilized in the cytosol. These patterns are typical for PTD-internalized cargos, reflecting their entrance via the endosomal route (27). 
Table I. Quantification of the internalization of SA and ST by Tat13-fused ligands.

\begin{tabular}{lccc}
\hline HRP-fused & Tat13-fused & $\begin{array}{c}\mathrm{K}_{\mathrm{D}} \\
(\mu \mathrm{M})\end{array}$ & $\begin{array}{c}\text { Quantification } \\
(\mathrm{pmol} / \mathrm{mg})\end{array}$ \\
\hline $\mathrm{SA}$ & $\mathrm{PEO}_{3}$-biotin & $10^{-8}$ & $26-72$ \\
$\mathrm{ST}$ & $\mathrm{PEO}_{3}$-biotin & $<1$ & $22-29$ \\
$\mathrm{ST}$ & Strep-tag II & 1 & $4.2-14$ \\
$\mathrm{SA}$ & Strep-tag II & 72 & $0.56-1.5$ \\
\hline
\end{tabular}

Internalized amounts of HRP were calculated in pmol of internalized SA-HRP or ST-HRP, normalized to the total amount of protein.

Notably, ST could also be efficiently internalized into cells by non-covalent binding to its ligand Strep-tag II, fused to the Tat13 PTD (Fig. 2A). This is remarkable, since the binding affinity between ST and Strep-tag II $\left(\mathrm{K}_{\mathrm{D}}=1 \mu \mathrm{M}\right)(23)$ is substantially lower than the affinity between $\mathrm{SA}$ and biotin $\left(K_{D}=10^{-14} \mathrm{M}\right)(17)$. HeLa cells treated with complexes of ST and Tat13-Strep-tag II were almost 100\% ST-positive (Fig. 2A) and exhibited the same intracellular distribution as observed for SA introduced by Tat13- $\mathrm{PEO}_{3}$-biotin (Fig. 2B).

Finally, the internalization of SA by Tat13-Strep-tag II $\left[\mathrm{K}_{\mathrm{D}}=72 \mu \mathrm{M}(28)\right]$ and ST by Tat13-PEO ${ }_{3}$-biotin $\left(\mathrm{K}_{\mathrm{D}}<1 \mu \mathrm{M}\right)$ was tested. Again, HeLa cells were almost $100 \%$ positive for the SA or ST cargos, respectively, after treatment with the corresponding complexes (Fig. 2A). Control-treated cells showed no cargo internalization (Fig. 2A). Taken together, these results demonstrate efficient internalization of both SA and ST by non-covalently bound PTDs.

Quantification of internalization. To estimate the efficiency of transmembrane delivery, horseradish peroxidase (HRP) fused to SA or ST was applied as cargo. HeLa cells were incubated with $1 \mu \mathrm{M}$ of SA-HRP or ST-HRP complexed with $2 \mu \mathrm{M}$ Tat13-fused ligands. Substantial enzymatic HRP activities were measured only in lysates of HeLa cells treated with SA-HRP or ST-HRP if complexed with the respective Tat13ligands (data not shown). SA-HRP and ST-HRP calibration curves were calculated to estimate the amount of Tat13mediated internalization. The internalization rates were quantified with respect to the amount of functional SA-HRP and ST-HRP in pmol, and normalized to the total amount of protein (Table I). The internalization efficiencies of ST-HRP by Tat13-Strep-tag II were lower than those of SA-HRP internalized by Tat13- $\mathrm{PEO}_{3}$-biotin, in line with the different ligand affinities (see above).

Ligand replacement by an intracellular target. Next, we tested whether a binding protein internalized by non-covalent binding to a PTD-fused ligand could be utilized to sequester an intracellular target by ligand replacement. As model systems, we employed SA and ST internalized via their ligand Strep-tag II linked to the Tat13 PTD (schematic model in Fig. 1). We then tested whether Tat13-Strep-tag II was replaced by intracellular biotin which binds to the same pocket as Strep-tag II, but with higher affinity $(22,23)$.
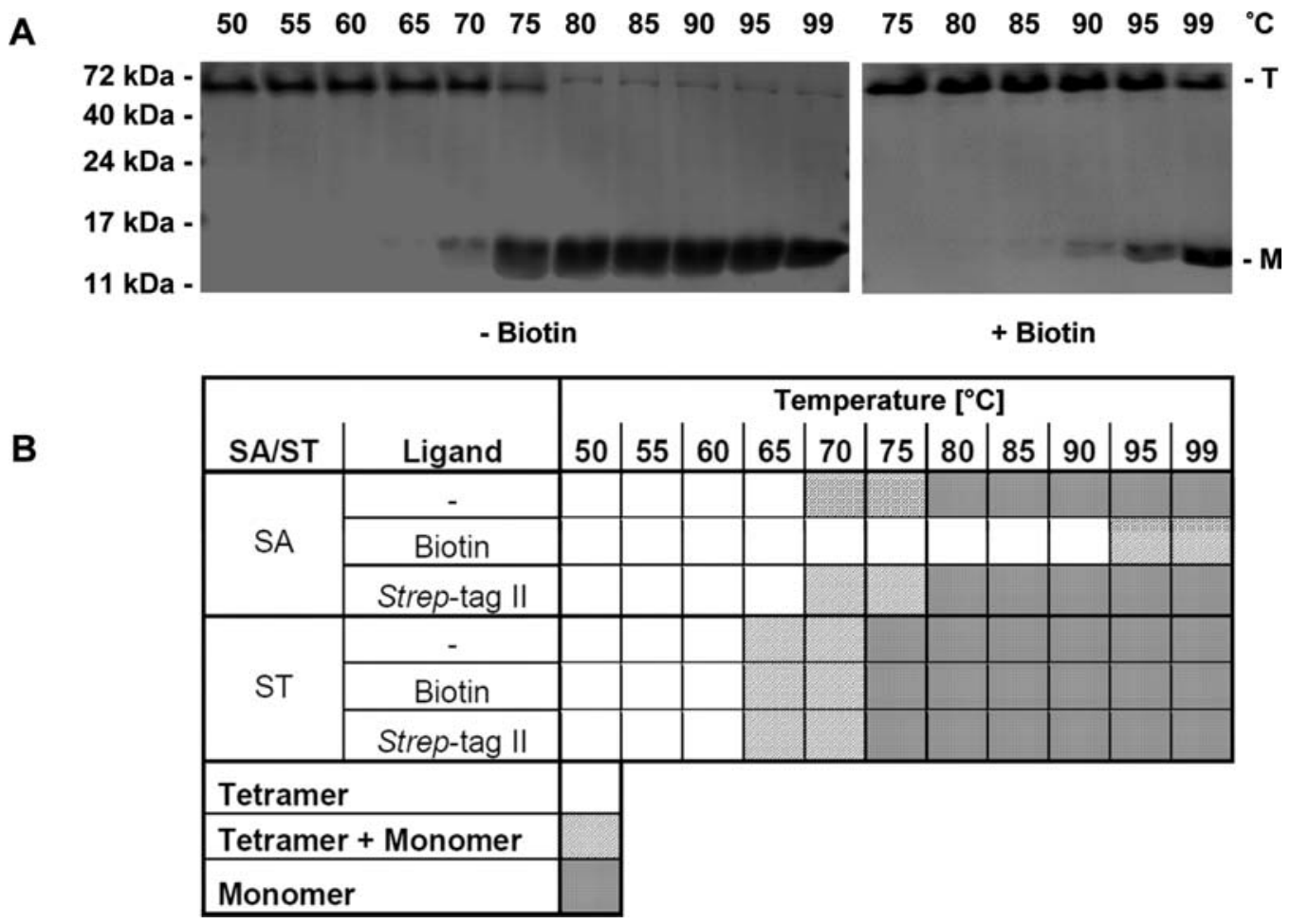

Figure 3. Thermal tetramer stability of SA and ST. (A) Exemplary SDS-PAGE analyses of Coomassie-stained SA in the absence of a ligand (left panel) or after addition of biotin (right panel). Tetrameric (T) and monomeric (M) states are indicated. (B) Compilation of thermal stabilities of SA and ST, either in the absence or presence of the ligands biotin and Strep-tag II. 
Tat13-SA

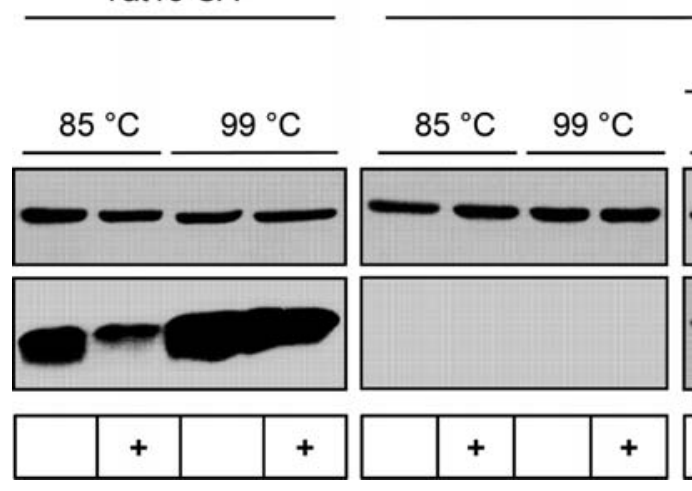

SA

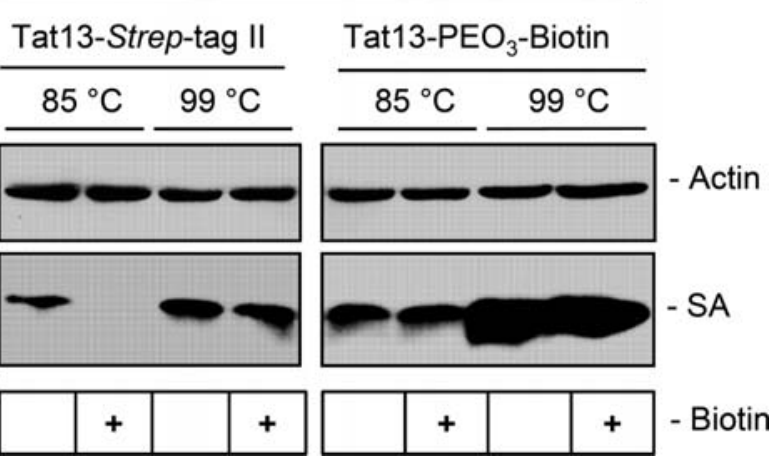

Figure 4. Intracellular replacement of Tat13-Strep-tag II on SA by biotin. Immunoblot analysis of monomeric SA. SA was internalized by covalently bound Tat13 or by non-covalently linked Tat13-Strep-tag II or Tat13-PEO -biotin. Actin, loading control.

First, it was necessary to investigate whether the ligandlinked PTDs might affect SA or ST tetramerization (24) which is required for building the binding pocket for biotin and Strep-tag II (29). SA and ST tetramers are extremely stable even in the presence of SDS and therefore can be detected on SDS-polyacrylamide gels $(30,31)$. Temperature-dependence of tetramer breakup was studied by heating and subsequent SDS-PAGE analyses. Up to a temperature of $65^{\circ} \mathrm{C}, \mathrm{SA}$ proteins were detectable solely in a tetrameric state (Fig. 3). The amino acid substitutions to convert SA into ST decreased the tetramer stability by $5^{\circ} \mathrm{C}$ (Fig. 3B). The pre-incubations of ST with biotin or Strep-tag II had no effect on the temperaturedependent ST tetramer breakup. Likewise, pre-incubation of SA with Strep-tag II peptides induced no changes in the temperature-dependent SA tetramer breakup. Notably, however, SA complexed with biotin showed an increased tetramer stability up to $90^{\circ} \mathrm{C}$ (Fig. 3), as previously reported (31). This increased tetramerization can be readily visualized by the strongly reduced amounts of monomeric SA at higher temperatures (Fig. 3A), thereby providing a readily accessible readout for the biotin/SA complex formation.

As a positive control for cellular assays (Fig. 4), HeLa cells were treated with $10 \mu \mathrm{M}$ Tat13-SA where the PTD is covalently linked to SA. After $2 \mathrm{~h}$, cells were extensively washed and, subsequently, $40 \mathrm{mM}$ biotin was added to the cells. Biotin-uptake was supported by means of the mammalian multivitamin transporter (32). After 2 additional hours, cell lysates were prepared and analyzed by immunoblotting. Addition of biotin to the cells resulted in a clear reduction of SA monomers at $85^{\circ} \mathrm{C}$ (Fig. 4), reflecting increased tetramer stability.

Next, HeLa cells were treated with complexes of $10 \mu \mathrm{M} \mathrm{SA}$ and $20 \mu \mathrm{M}$ Tat13-Strep-tag II. As observed for the positive control, intracellular SA tetramers were thermally stabilized by the addition of biotin to the cells, as indicated by the decreased amount of SA monomers at $85^{\circ} \mathrm{C}$ (Fig. 4). These findings show that the same binding pocket which has been used for SA internalization by a non-covalently bound ligand can be used for intracellular sequestration of an higher affinity target.

As further control, we employed Tat13- $\mathrm{PEO}_{3}$-biotin-internalized SA. Since Tat13- $\mathrm{PEO}_{3}$-biotin, as a biotin-derivative, already strongly binds to the SA pocket, it would be expected that addition of biotin to the cells does not further increase thermal stabilities. Indeed, and in contrast to Tat13-SA or SA introduced by Tat13-Strep-tag II, the Tat13-PEO - $\mathrm{P}_{3}$-biotininternalized SA was not thermally stabilized by the addition of biotin to the cells (Fig. 4).

Taken together, these results strongly suggest that intracellular biotin induced the thermal stabilization of SA tetramers by replacing Tat13-Strep-tag II on SA. Thus, the internalization of a binding protein by a low affinity ligand fused to a PTD is feasible and can be utilized for subsequent ligand replacement inside the cell (Fig. 1).

\section{Discussion}

The delivery of molecules into cells poses a major problem that has to be solved for the development of therapeutic agents acting on intracellular targets. Cargos which cannot penetrate cellular membranes by themselves due to their biophysical properties can achieve cell membrane permeability by fusion to PTDs (11). Notably, however, the design and generation of PTD-fused proteins can result in reduced expression and purification levels and/or in altered biophysical properties of cargos (10). Therefore, a transporter system for the transmembrane delivery of non-modified cargos was developed. It aimed at a PTD-mediated internalization of a non-covalently linked protein which can capture an intracellular target molecule by ligand exchange.

The components of this model system consisted of: i) SA, a model binding protein containing a binding pocket for its intracellular target biotin, ii) the PTD-fused ligand Strep-tag II, a cell-permeable transporter which more weakly interacts with the same pocket, and iii) biotin, which should replace the PTD-Strep-tag II ligand at the intracellular level due to its higher binding affinity to SA. Both immunoblot and immunofluorescence analyses demonstrated that Tat13-Streptag II mediated the successful transmembrane delivery of non-covalently bound SA. Moreover, supplemented biotin displaced the Tat13-Strep-tag II ligand as confirmed by an increased thermal tetramer stability of internalized SA.

The internalization of SA and ST by non-covalent linkage to Tat13-fused ligands was found to be highly efficient in that almost $100 \%$ of the treated cells were positive for SA 
or ST. Similar efficiencies have been reported for the use of covalently linked PTDs (33). The internalization efficiency by Tat13-fused Strep-tag II was further quantified using functionally active HRP fused to SA. It was lower $(\sim 1$ pmol per mg of total cellular protein) than the reported efficiency of the internalization of FITC-SA by Tat11-biotin $(23 \mathrm{pmol} / \mathrm{mg})$ (26). This could be explained by the $>2$-fold increased MW of SA-HRP ( 134 kDa) above FITC-SA $(\sim 60 \mathrm{kDa})$ and by the law of mass action.

The system developed in the present work provides some important advantages over existing transporter systems. Firstly, it allows the delivery of a non-modified cargo and avoids potential problems associated with the covalent linkage of a PTD, such as reduced expression and purification yields and impairment of the biophysical properties of the cargo (10). Secondly, in contrast to previously designed transmembrane delivery systems, such as Tat11-SA for biotinylated molecules $(27,34)$ or Tat11-fused protein A for antibodies $(35,36)$, the transporter employed here is less bulky since it only consists of low-MW components: a PTD and a small ligand. Thirdly, since the active site of the internalized cargo is reversibly occupied by a PTD-fused ligand, only molecules possessing a higher affinity to the cargo should be able to displace the ligand which increases specificity.

Taken together, the findings of this study indicate that, in principle, various engineered binding molecules, such as anticalins $(5,37)$ (Fig. 1) or peptide aptamers $(38)$, could be tailored for the intracellular sequestration of a given target molecule by similar strategies. For example, specific binding proteins could be internalized by a PTD-fused ligand which is derived from a natural interaction partner of the binding protein, but exhibits reduced binding affinity due to chemical modifications. After PTD-mediated internalization, this weakly bound ligand should be displaced by the higher affinity intracellular target, as shown here for the replacement of PTD-Strep-tag II by biotin.

\section{Acknowledgements}

The authors are grateful to P.S. Stayton (University of Washington, WA, USA) for the core SA expression vector, A. Hunziker (Sequencing Core Facility, German Cancer Research Center) for DNA-sequencing, and R. Pipkorn (Peptide Synthesis Core Facility, German Cancer Research Center) for peptide synthesis.

\section{References}

1. Leader B, Baca QJ and Golan DE: Protein therapeutics: a summary and pharmacological classification. Nat Rev Drug Discov 7: 21-39, 2008.

2. Groner B, Hartmann C and Wels W: Therapeutic antibodies. Curr Mol Med 4: 539-547, 2004.

3. Holliger P and Hudson PJ: Engineered antibody fragments and the rise of single domains. Nat Biotechnol 23: 1126-1136, 2005.

4. Binz HK, Amstutz P and Pluckthun A: Engineering novel binding proteins from nonimmunoglobulin domains. Nat Biotechnol 23: 1257-1268, 2005.

5. Schlehuber S and Skerra A: Anticalins as an alternative to antibody technology. Expert Opin Biol Ther 5: 1453-1462, 2005.

6. Frankel $\mathrm{AD}$ and Pabo CO: Cellular uptake of the tat protein from human immunodeficiency virus. Cell 55: 1189-1193, 1988 .
7. Green M and Loewenstein PM: Autonomous functional domains of chemically synthesized human immunodeficiency virus tat trans-activator protein. Cell 55: 1179-1188, 1988.

8. Fawell S, Seery J, Daikh Y, et al: Tat-mediated delivery of heterologous proteins into cells. Proc Natl Acad Sci USA 91: 664-668, 1994.

9. Vives E, Brodin P and Lebleu B: A truncated HIV-1 Tat protein basic domain rapidly translocates through the plasma membrane and accumulates in the cell nucleus. J Biol Chem 272: 16010-16017, 1997

10. Honda A, Moosmeier MA and Dostmann WR: Membranepermeable cygnets: rapid cellular internalization of fluorescent cGMP-indicators. Front Biosci 10: 1290-1301, 2005.

11. Dietz GP and Bahr M: Delivery of bioactive molecules into the cell: the Trojan horse approach. Mol Cell Neurosci 27: 85-131, 2004.

12. Gump JM and Dowdy SF: TAT transduction: the molecular mechanism and therapeutic prospects. Trends Mol Med 13: 443-448, 2007.

13. Trehin R and Merkle HP: Chances and pitfalls of cell penetrating peptides for cellular drug delivery. Eur J Pharm Biopharm 58: 209-223, 2004.

14. Borghouts C, Kunz C and Groner B: Current strategies for the development of peptide-based anti-cancer therapeutics. J Pept Sci 11: 713-726, 2005.

15. Park J, Ryu J, Jin LH, et al: 9-polylysine protein transduction domain: enhanced penetration efficiency of superoxide dismutase into mammalian cells and skin. Mol Cells 13: 202-208, 2002.

16. Jin LH, Bahn JH, Eum WS, et al: Transduction of human catalase mediated by an HIV-1 TAT protein basic domain and arginine-rich peptides into mammalian cells. Free Radic Biol Med 31: 1509-1519, 2001.

17. Rothbard JB, Garlington S, Lin Q, et al: Conjugation of arginine oligomers to cyclosporin A facilitates topical delivery and inhibition of inflammation. Nat Med 6: 1253-1257, 2000.

18. Green NM: Avidin and streptavidin. Methods Enzymol 184: 51-67, 1990.

19. Mi Z, Mai J, Lu X and Robbins PD: Characterization of a class of cationic peptides able to facilitate efficient protein transduction in vitro and in vivo. Mol Ther 2: 339-347, 2000.

20. Mai JC, Shen H, Watkins SC, Cheng T and Robbins PD: Efficiency of protein transduction is cell type-dependent and is enhanced by dextran sulfate. J Biol Chem 277: 30208-30218, 2002.

21. Laitinen OH, Nordlund HR, Hytonen VP and Kulomaa MS: Brave new (strept)avidins in biotechnology. Trends Biotechnol 25: 269-277, 2007.

22. Laitinen OH, Hytonen VP, Nordlund HR and Kulomaa MS: Genetically engineered avidins and streptavidins. Cell Mol Life Sci 63: 2992-3017, 2006.

23. Schmidt TG and Skerra A: The Strep-tag system for one-step purification and high-affinity detection or capturing of proteins. Nat Protoc 2: 1528-1535, 2007.

24. Voss S and Skerra A: Mutagenesis of a flexible loop in streptavidin leads to higher affinity for the Strep-tag II peptide and improved performance in recombinant protein purification. Protein Eng 10: 975-982, 1997.

25. Schmidt TG and Skerra A: One-step affinity purification of bacterially produced proteins by means of the 'Strep tag' and immobilized recombinant core streptavidin. J Chromatogr A 676: 337-345, 1994.

26. El-Andaloussi S, Jarver P, Johansson HJ and Langel U: Cargo dependent cytotoxicity and delivery efficacy of cellpenetrating peptides: a comparative study. Biochem J 407: 285-292 2007.

27. Rinne J, Albarran B, Jylhava J, et al: Internalization of novel non-viral vector TAT-streptavidin into human cells. BMC Biotechnol 7: 1, 2007.

28. Schmidt TG, Koepke J, Frank R and Skerra A: Molecular interaction between the Strep-tag affinity peptide and its cognate target, streptavidin. J Mol Biol 255: 753-766, 1996.

29. Sano T and Cantor CR: Intersubunit contacts made by tryptophan 120 with biotin are essential for both strong biotin binding and biotin-induced tighter subunit association of streptavidin. Proc Natl Acad Sci USA 92: 3180-3184, 1995.

30. Waner MJ, Navrotskaya I, Bain A, Oldham ED and Mascotti DP: Thermal and sodium dodecylsulfate induced transitions of streptavidin. Biophys J 87: 2701-2713, 2004. 
31. Bayer EA, Ehrlich-Rogozinski S and Wilchek M: Sodium dodecyl sulfate-polyacrylamide gel electrophoretic method for assessing the quaternary state and comparative thermostability of avidin and streptavidin. Electrophoresis 17: 1319-1324, 1996.

32. Prasad PD and Ganapathy V: Structure and function of mammalian sodium-dependent multivitamin transporter. Curr Opin Clin Nutr Metab Care 3: 263-266, 2000.

33. Lea NC, Buggins AG, Orr SJ, Mufti GJ and Thomas NS: High efficiency protein transduction of quiescent and proliferating primary hematopoietic cells. J Biochem Biophys Methods 55: 251-258, 2003.

34. Albarran B, To R and Stayton PS: A TAT-streptavidin fusion protein directs uptake of biotinylated cargo into mammalian cells. Protein Eng Des Sel 18: 147-152, 2005.
35. Mie M, Takahashi F, Funabashi H, Yanagida Y, Aizawa M and Kobatake E: Intracellular delivery of antibodies using TAT fusion protein A. Biochem Biophys Res Commun 310: 730-734, 2003.

36. Mie M, Mori K, Funabashi H and Kobatake E: Delivery of antibody-captured proteins into living cells using PTD-fused protein A. Biotechnol Lett 28: 1209-1214, 2006.

37. Skerra A: Anticalins as alternative binding proteins for therapeutic use. Curr Opin Mol Ther 9: 336-344, 2007

38. Bickle MB, Dusserre E, Moncorge O, Bottin H and Colas P: Selection and characterization of large collections of peptide aptamers through optimized yeast two-hybrid procedures. Nat Protoc 1: 1066-1091, 2006. 